\title{
Predictors of increased body weight and waist circumference for middle-aged adults
}

\author{
Robert J Maclnnis ${ }^{1,2, *}$, Allison M Hodge ${ }^{1}$, Helen G Dixon ${ }^{3}$, Anna Peeters ${ }^{4,5}$, \\ Lucinda EA Johnson ${ }^{1}$, Dallas R English ${ }^{1,2}$ and Graham G Giles ${ }^{1,2}$ \\ ${ }^{1}$ Cancer Epidemiology Centre, Cancer Council Victoria, 1 Rathdowne Street, Carlton, Victoria 3053, Australia: \\ ${ }^{2}$ Centre for Molecular, Environmental, Genetic and Analytic Epidemiology, School of Population Health, \\ The University of Melbourne, Carlton, Victoria, Australia: ${ }^{3}$ Centre for Behavioural Research in Cancer, Cancer \\ Council Victoria, Carlton, Victoria, Australia: ${ }^{4}$ Obesity \& Population Health Unit, Baker IDI Heart and Diabetes \\ Institute, Melbourne, Victoria, Australia: ${ }^{5}$ School of Population Health and Preventive Medicine, Monash \\ University, Melbourne, Victoria, Australia
}

Submitted 17 August 2012: Final revision received 28 February 2013: Accepted 8 March 2013: First published online 1 May 2013

\begin{abstract}
Objective: To identify predictors of increased adiposity for different measures of adiposity.

Design: Prospective cohort study, the Melbourne Collaborative Cohort Study (MCCS), with data at baseline (1990-1994) and wave 2 (2003-2007).

Setting: Participants recruited from the community.

Subjects: Australian-born participants ( $n$ 5879) aged 40 to 69 years who were not current smokers and who were free from common chronic diseases at recruitment. At baseline and at wave 2, weight and waist circumference were measured; while demographic and lifestyle variables were obtained at baseline via structured interviews.

Results: Participants who reported any recreational physical activity at baseline had lower weight and smaller waist circumference at wave 2 than those who did not, particularly for younger participants and for vigorous physical activity. Walking for leisure was not associated, and greater physical activity at work was associated, with greater adiposity measures at wave 2 . A diet low in carbohydrates and fibre, but high in fat and protein, predicted greater weight and waist circumference at wave 2. Participants were less likely to have elevated weight or waist circumference at wave 2 if they consumed low to moderate amounts of alcohol.

Conclusions: Our findings indicate that promoting vigorous physical activity, encouraging a diet high in carbohydrate and fibre but low in fat and protein, and limiting alcohol intake could be promising approaches for preventing obesity in middle-aged adults. Similar interventions should successfully address the management of both weight and waist circumference, as they were predicted by similar factors.
\end{abstract}

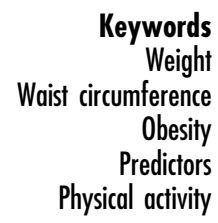

Understanding the individual-level predictors of weight and waist circumference gain is critical, both to identify individuals most at risk and to allow targeting of the behavioural risk factors likely to contribute the most to overweight and obesity. While consensus is forming around the role of specific dietary elements and markers of physical activity and inactivity associated with increased weight gain $^{(1-3)}$, few studies have analysed predictors of increasing waist circumference ${ }^{(4-7)}$ and the majority of these have focused solely on dietary factors.

Waist circumference is a better indicator of excess visceral adiposity $^{(8)}$, which tends to be more closely associated with metabolic abnormalities and some cancers than overall adiposity as assessed by $\mathrm{BMI}^{(3)}$. Further, there is evidence that waist circumference is increasing at a faster rate than $\mathrm{BMI}^{(7,9)}$, suggesting that there may be differential drivers of BMI and waist circumference. A recent analysis of cohorts in the European Prospective Investigation into Cancer and Nutrition suggested that specific dietary elements may predict changes in waist circumference independent of changes in $\mathrm{BMI}^{(5)}$. However, no studies have simultaneously analysed the association between a wide range of behavioural factors and changes in weight and waist circumference.

The predictors of weight gain may differ from the predictors of weight loss ${ }^{(10)}$. The health benefits for overweight individuals of losing weight compared with maintaining a stable weight are still unknown ${ }^{(11)}$. Exploring predictors 
of weight loss, in particular for elderly populations, is problematic as weight loss may be unintentional due to an undiagnosed disease ${ }^{(12)}$.

We evaluated potential predictors of stable and increased weight and waist circumference using a prospective cohort study in which all participants were directly measured at baseline recruitment and at a later face-to face examination (mean 11.7 years after baseline), which we refer to as wave 2 . We focused on potentially modifiable predictors such as physical activity (both leisure time and work related), alcohol consumption and nutrient intake for participants who were not current smokers and were free from common chronic diseases at baseline.

\section{Participants and methods}

\section{Participants}

The Melbourne Collaborative Cohort Study (MCCS) is a prospective cohort study comprising 17045 men and 24469 women, aged between 27 and 75 years at baseline (99.3\% aged 40 to 69 years). Participants were recruited from the Melbourne metropolitan area between 1990 and 1994 using the electoral rolls for direct invitations, advertisements and community announcements. Southern European migrants were oversampled at the time of recruitment to help increase the variability of dietary and non-dietary risk factors. Further details of the study have been published elsewhere ${ }^{(13)}$. The study was conducted according to the guidelines laid down in the Declaration of Helsinki and all procedures involving participants were approved by the Cancer Council Victoria's Human Research Ethics Committee. Written informed consent was obtained from all participants.

A randomly selected total of 11994 participants was targeted for attendance at wave 2 between 2003 and 2007. Participants were excluded if, at baseline, they were aged less than 40 years or over 69 years $(n 7)$, had energy intakes in the extreme centiles of the sex-specific distributions ( $n$ 244), were missing values of weight or waist circumference ( $n 9)$, had a pre-existing disease (diabetes, cancer, angina, stroke or heart attack; $n$ 1500) or were current smokers (to avoid possible weight changes due to quitting smoking; $n$ 1168). After these exclusions, 9066 participants were eligible.

\section{Ascertainment of cancer status and vital status}

Cancer cases were identified from notifications to the Victorian Cancer Registry of diagnoses of invasive cancer (International Classification of Diseases 9th revision rubric 140-208 or 10th revision rubric C00-C99). Vital status and place of residence were obtained from electoral rolls, electronic phone books and death records until 2010.

\section{Direct physical measurements}

All participants attended a study centre at baseline, where physical measurements and blood sampling were performed.
Height, weight and waist circumference were measured according to standard procedures ${ }^{(14)}$. Weight and waist circumference were measured similarly at wave 2 . Heart rate was measured at baseline using Dinamap automatic monitors (see MacInnis et al. ${ }^{(15)}$ for further details).

\section{Questionnaire data and constructed variables}

Participants' residential postcodes at baseline were used to assign them to a quintile of socio-economic status (SES) according to the Index of Relative Socio-economic Advantage and Disadvantage that was obtained from the Australian Bureau of Statistics census-based SocioEconomic Indexes for Areas ${ }^{(16)}$.

A structured interview schedule was used to obtain information on country of birth, smoking ('Have you ever smoked at least seven cigarettes a week for at least a year?' and 'Do you now smoke at least seven cigarettes a week?'), highest level of education and self-reported history of diabetes, angina, stroke and heart attack.

Questions relating to frequency of walking (for recreation or exercise), vigorous exercise (exercise 'making you sweat or feel out of breath, and includes such activities as swimming, tennis, netball, athletics and running') and less-vigorous exercise (exercise 'which did not make you sweat or feel out of breath and includes such activities as bike riding, dancing, etc.') over the last 6 months were asked at baseline. These questions were obtained from the Risk Factor Prevalence Study conducted by the National Heart Foundation and Australian Institute of Health ${ }^{(17)}$. The reported frequency for each question was coded as follows: 0 (none), 1.5 (one or two times per week) or 4 (three or more times per week). Walking and less-vigorous exercise frequencies were added together along with two times the frequency of vigorous exercise to generate a physical activity score for each person. This score was then grouped based on approximate quartiles $(0,>0-3,>3-4,>4)$. Two further questions were also asked about how much time participants spent in moderate to heavy physical exertion at work and at home.

Dietary data were collected at baseline using a selfadministered 121-item FFQ specifically developed for the study $^{(18)}$. Nutrient intakes were computed from the FFQ based on the NUTTAB95 nutrient composition data ${ }^{(19)}$. Intakes of protein, fat, total carbohydrates and fibre were analysed as their proportion of total energy intake, with each gram assumed to contain 17, 37, 16 and $1 \mathrm{~kJ}$ of total energy, respectively ${ }^{(20)}$. Alcohol intake data were collected at baseline using beverage-specific questions of frequency and quantity.

\section{Statistical analysis}

Multivariable linear regression was used for cross-sectional analyses and to predict weight and waist circumference at wave 2. Analyses for each predictor were adjusted for possible confounders at baseline identified a priori, 
including baseline measures of weight (or waist circumference), sex, age, physical activity (pseudocontinuous), alcohol intake (none, 0·1-19·9, 20·0-39·9, $\geq 40 \cdot 0 \mathrm{~g} / \mathrm{d}$ ), educational attainment (primary school/some high or technical school, completed high or technical school, degree/diploma), smoking status (never, past), marital status (married/de facto, single/divorced/widowed/ separated), SES (pseudo-continuous, based on approximate quintiles) and total dietary energy intake $(\mathrm{kJ} / \mathrm{d})$. Additional sub-analyses were also performed, including: tests for interactions between age, sex and predictor variables; further adjustment for duration since baseline; analyses restricted to participants without disease onset before wave 2 (cancer, heart attack, stroke, angina and diabetes); and analyses excluding those who reported having hypertension at baseline.

Statistical analyses were performed using the statistical software package Stata version $12 \cdot 0$.

\section{Results}

After baseline, 656 of the 9066 eligible people died before wave 2 was completed and a further 1894 declined to attend for physical examination at wave 2 . Twenty-one of the people who attended wave 2 were excluded as they had no weight or waist circumference measurements collected at wave 2, leaving 6495 (72\%). A further 616 participants who lost $5 \mathrm{~kg}$ or more in weight between baseline and wave 2 were excluded because the predictors of loss may differ from those of maintenance or gain. This left 5879 participants in the final analysis.

On average, non-participants at wave 2 (which included current smokers at baseline) were more likely to be older (56 v. 54 years old), male ( $44 \% v .36 \%$ ), born in Southern Europe $(31 \% v$. 18\%), less educated (36\%v $v$ $51 \%$ completed high school), not married ( $32 \% v .27 \%$ ), heavier (males $81 v .80 \mathrm{~kg}$, females $69 v .67 \mathrm{~kg}$ ) and to have larger waist circumference (males 94 v. $92 \mathrm{~cm}$, females $81 v .78 \mathrm{~cm}$ ) than participants at baseline.

At wave 2 , on average 11.7 years since baseline, participants were on average $3 \cdot 3 \mathrm{~kg}$ heavier and had $8.0 \mathrm{~cm}$ larger waist circumferences. Additional descriptive characteristics of the participants are shown in Table 1.

Table 2 shows the cross-sectional associations between both weight and waist circumference at baseline and the various exposures. Measures of weight and waist circumference were greater for men and older participants at baseline, while many of the modifiable baseline exposures had positive (protein and fat intake) or negative (exercise, walking, low alcohol, carbohydrate and fibre intakes) associations with weight and waist circumference at baseline.

Table 3 shows the associations between both weight and waist circumference at wave 2 and the various baseline exposures. Measures of weight and waist circumference were higher for women and younger participants at wave 2 .
Also, the longer the duration since baseline, the greater the weight and waist circumference were at wave 2 . Participants born in Southern Europe had a larger waist circumference at wave 2 .

Participants who had completed high school or above had a lower waist circumference but not weight at wave 2 compared with those who had not. Weight and waist circumference at wave 2 were greater for people living in more disadvantaged areas. Married and de facto persons had on average lower weight at wave 2 than single, divorced or widowed people. Notably, greater waist circumferences at wave 2 were observed for divorced compared with married participants.

Positive associations were observed between waist circumference at wave 2 and baseline cardiovascular measures (systolic pressure, diastolic pressure, pulse rate) in contrast to the null associations with weight at wave 2 .

Those who reported participating in recreational exercise at baseline had lower weight and smaller waist circumference at baseline and at wave 2 than those who did not. The associations strengthened with exercise intensity and frequency. The association with exercise was mainly restricted to younger participants (see Tables 4 and 5). On the other hand, moderate or heavy activity at work was positively associated with waist circumference at wave 2 , even after adjustment for recreational exercise. Despite the fact that those participants who walked three times or more per week had lower baseline weight and smaller waist circumference, there was no discernible association between leisure-time walking and future weight or waist circumference.

There was no notable association observed with smoking status. Participants were less likely to have an elevated weight or waist circumference at wave 2 if they consumed low to moderate amounts of alcohol.

Baseline energy intake showed no association with future weight or waist circumference, but a higher proportional consumption of protein and fat at baseline was positively associated with both weight and waist circumference at wave 2, while carbohydrate and fibre consumption were negatively associated with waist circumference but not weight at wave 2 . As with exercise, the associations with these nutrients were strongest for younger participants (see Tables 4 and 5).

Analyses restricted to participants without disease onset before wave 2 (cancer, heart attack, stroke, angina and diabetes) gave similar results to the main analyses (data not shown) and associations remained virtually unchanged after further adjustment for duration of follow-up time since baseline (data not shown). Analyses excluding those who reported having hypertension at baseline (1031 people) gave similar results to the main analyses (data not shown), except that systolic $(\beta$ coefficient $=-0 \cdot 11, \mathrm{sE}=0 \cdot 05, P=0 \cdot 04)$ and diastolic $(\beta$ coefficient $=-0 \cdot 16, \mathrm{sE}=0 \cdot 08, P=0 \cdot 04)$ blood pressure were inversely associated with weight at wave 2 . 
Table 1 Characteristics of participants stratified by sex: adults with data at baseline (1990-1994) and wave 2 (2003-2007), Melbourne Collaborative Cohort Study

\begin{tabular}{|c|c|c|c|c|}
\hline & \multicolumn{2}{|c|}{ Male } & \multicolumn{2}{|c|}{ Female } \\
\hline & Mean or $n$ & $\%$ or SD & Mean or $n$ & $\%$ or SD \\
\hline$N$ & \multicolumn{2}{|c|}{2128} & \multicolumn{2}{|c|}{3751} \\
\hline Age at baseline (years) & $53 \cdot 5$ & $8 \cdot 4$ & $53 \cdot 6$ & $8 \cdot 2$ \\
\hline Duration of follow-up (years) & $11 \cdot 7$ & 1.5 & $11 \cdot 7$ & $1 \cdot 5$ \\
\hline Weight at baseline $(\mathrm{kg})$ & $80 \cdot 2$ & $11 \cdot 1$ & $67 \cdot 2$ & $11 \cdot 3$ \\
\hline Weight at wave $2(\mathrm{~kg})$ & $82 \cdot 9$ & $12 \cdot 7$ & $70 \cdot 7$ & $13 \cdot 4$ \\
\hline Waist at baseline $(\mathrm{cm})$ & $91 \cdot 7$ & $9 \cdot 4$ & $78 \cdot 0$ & $10 \cdot 9$ \\
\hline Waist at wave $2(\mathrm{~cm})$ & $98 \cdot 1$ & $10 \cdot 6$ & $87 \cdot 0$ & $12 \cdot 6$ \\
\hline \multicolumn{5}{|l|}{ Weight change at wave $2(\%)$} \\
\hline Within $5 \mathrm{~kg}$ of baseline weight & 1554 & $73 \cdot 0$ & 2555 & $68 \cdot 1$ \\
\hline Gained $5-9.9 \mathrm{~kg}$ & 413 & $19 \cdot 4$ & 747 & $19 \cdot 9$ \\
\hline Gained $10 \mathrm{~kg}$ or more & 161 & $7 \cdot 6$ & 449 & $12 \cdot 0$ \\
\hline \multicolumn{5}{|l|}{ Country of birth (\%) } \\
\hline Australia/New Zealand/UK & 1722 & $80 \cdot 9$ & 3097 & $82 \cdot 6$ \\
\hline Greece/Italy & 406 & $19 \cdot 1$ & 654 & $17 \cdot 4$ \\
\hline \multicolumn{5}{|l|}{ Highest level of education (\%) } \\
\hline Primary/some high/technical school & 828 & $38 \cdot 9$ & 2079 & $55 \cdot 4$ \\
\hline Completed high school & 554 & $26 \cdot 0$ & 706 & $18 \cdot 8$ \\
\hline Completed tertiary degree/diploma & 746 & $35 \cdot 1$ & 966 & $25 \cdot 8$ \\
\hline \multicolumn{5}{|l|}{ Smoking status (\%) } \\
\hline Never & 1187 & $55 \cdot 8$ & 2801 & $74 \cdot 7$ \\
\hline Former & 941 & $44 \cdot 2$ & 950 & $25 \cdot 3$ \\
\hline \multicolumn{5}{|l|}{ Marital status (\%) } \\
\hline Married & 1644 & $80 \cdot 0$ & 2501 & $69 \cdot 2$ \\
\hline Single & 184 & $9 \cdot 0$ & 322 & $8 \cdot 9$ \\
\hline Divorced & 97 & $4 \cdot 7$ & 343 & $9 \cdot 5$ \\
\hline De facto & 61 & $3 \cdot 0$ & 71 & $2 \cdot 0$ \\
\hline Widowed & 38 & 1.9 & 290 & $8 \cdot 0$ \\
\hline Separated & 32 & $1 \cdot 6$ & 85 & $2 \cdot 4$ \\
\hline \multicolumn{5}{|c|}{ Index of Relative Socio-economic Disadvantage (\%) } \\
\hline 1st quintile (most disadvantaged) & 222 & $10 \cdot 5$ & 450 & $12 \cdot 0$ \\
\hline 2nd quintile & 375 & $17 \cdot 7$ & 663 & $17 \cdot 7$ \\
\hline 3rd quintile & 389 & $18 \cdot 4$ & 673 & $18 \cdot 0$ \\
\hline 4th quintile & 466 & $22 \cdot 0$ & 822 & $22 \cdot 0$ \\
\hline 5th quintile (least disadvantaged) & 667 & $31 \cdot 5$ & 1132 & $30 \cdot 3$ \\
\hline Height $(\mathrm{cm})$ & $173 \cdot 5$ & $7 \cdot 2$ & $160 \cdot 8$ & $6 \cdot 5$ \\
\hline Energy intake (MJ/d) & $10 \cdot 5$ & $3 \cdot 2$ & $8 \cdot 6$ & $2 \cdot 8$ \\
\hline Protein (\% of energy) & 18 & 3 & 19 & 3 \\
\hline Fat (\% of energy) & 33 & 5 & 33 & 6 \\
\hline Carbohydrates (\% of energy) & 42 & 7 & 44 & 7 \\
\hline Fibre (\% of energy) & $0 \cdot 3$ & $0 \cdot 1$ & 0.4 & $0 \cdot 1$ \\
\hline Diastolic blood pressure $(\mathrm{mmHg})$ & $80 \cdot 1$ & $10 \cdot 5$ & $71 \cdot 9$ & $10 \cdot 6$ \\
\hline Systolic blood pressure $(\mathrm{mmHg})$ & $136 \cdot 3$ & $16 \cdot 5$ & $132 \cdot 0$ & $17 \cdot 9$ \\
\hline Pulse (beats/min) & $65 \cdot 9$ & $9 \cdot 5$ & $69 \cdot 7$ & $9 \cdot 1$ \\
\hline \multicolumn{5}{|l|}{ Total alcohol (\%) } \\
\hline Lifetime abstainers & 345 & $16 \cdot 2$ & 1366 & $36 \cdot 4$ \\
\hline $0.1-19.9 \mathrm{~g} / \mathrm{d}$ & 1025 & $48 \cdot 2$ & 1966 & $52 \cdot 4$ \\
\hline $20 \cdot 0-39 \cdot 9 \mathrm{~g} / \mathrm{d}$ & 448 & $21 \cdot 1$ & 332 & $8 \cdot 9$ \\
\hline$\geq 40.0 \mathrm{~g} / \mathrm{d}$ & 310 & $14 \cdot 6$ & 87 & $2 \cdot 3$ \\
\hline \multicolumn{5}{|l|}{ Walking (\%) } \\
\hline None & 904 & $42 \cdot 5$ & 1391 & $37 \cdot 1$ \\
\hline 1-2 times/week & 425 & $20 \cdot 0$ & 846 & $22 \cdot 6$ \\
\hline$\geq 3$ times/week & 799 & $37 \cdot 6$ & 1514 & $40 \cdot 4$ \\
\hline Exercise* $(\%)^{*}$ & & & & \\
\hline None & 901 & $42 \cdot 3$ & 1758 & $46 \cdot 9$ \\
\hline Less vigorous only ( $\geq$ once/week) & 546 & $25 \cdot 7$ & 1061 & $28 \cdot 3$ \\
\hline Vigorous ( $\geq$ once/week) & 681 & $32 \cdot 0$ & 932 & $24 \cdot 9$ \\
\hline Physical activity score (\%) & & & & \\
\hline 0 & 392 & $18 \cdot 4$ & 717 & $19 \cdot 1$ \\
\hline $1-3$ & 420 & $19 \cdot 7$ & 787 & $21 \cdot 0$ \\
\hline $4-5$ & 644 & $30 \cdot 3$ & 1334 & $35 \cdot 6$ \\
\hline$\geq 6$ & 672 & $31 \cdot 6$ & 913 & $24 \cdot 3$ \\
\hline Moderate/heavy activity at work (\%) & & & & \\
\hline None at all & 1816 & $85 \cdot 3$ & 3450 & $92 \cdot 0$ \\
\hline$\geq 1 \mathrm{~h} /$ week & 312 & $14 \cdot 7$ & 301 & $8 \cdot 0$ \\
\hline Moderate/heavy activity at home (\%) & & & & \\
\hline None at all & 1388 & $65 \cdot 2$ & 2470 & $65 \cdot 9$ \\
\hline$\geq 1 \mathrm{~h} /$ week & 740 & $34 \cdot 8$ & 1281 & $34 \cdot 2$ \\
\hline
\end{tabular}

${ }^{*}$ Participants in the vigorous group may have also reported doing less vigorous exercise. 
Table 2 Associations of potential predictors of weight and waist circumference at baseline*: adults with data at baseline (1990-1994) and wave 2 (2003-2007), Melbourne Collaborative Cohort Study

\begin{tabular}{|c|c|c|c|c|c|c|}
\hline & \multicolumn{3}{|c|}{ Weight at baseline (kg) } & \multicolumn{3}{|c|}{ Waist circumference at baseline $(\mathrm{cm})$} \\
\hline & $\beta$ & SE & $P$ value & $\beta$ & SE & $P$ value \\
\hline \multicolumn{7}{|l|}{ Sex } \\
\hline Male & 0 & Ref. & & 0 & Ref. & \\
\hline Female & $-4 \cdot 66$ & $0 \cdot 41$ & $<0.001$ & $-11 \cdot 64$ & 0.39 & $<0.001$ \\
\hline Age at baseline attendance (per 10 years) & 0.50 & $0 \cdot 17$ & 0.004 & 1.92 & $0 \cdot 16$ & $<0.001$ \\
\hline \multicolumn{7}{|l|}{ Country of birth } \\
\hline Australia/New Zealand/UK & 0 & Ref. & & 0 & Ref. & \\
\hline Greece/ltaly & $1 \cdot 82$ & $0 \cdot 20$ & $<0.001$ & $2 \cdot 15$ & $0 \cdot 19$ & $<0.001$ \\
\hline Follow-up time (per year) & 0.26 & $0 \cdot 11$ & 0.02 & $0 \cdot 30$ & $0 \cdot 10$ & 0.004 \\
\hline \multicolumn{7}{|l|}{ Education level } \\
\hline Primary, some high/technical school & 0 & Ref. & & 0 & Ref. & \\
\hline Completed high school & $-1 \cdot 13$ & $0 \cdot 37$ & 0.002 & $-1 \cdot 27$ & 0.35 & $<0.001$ \\
\hline Completed tertiary degree/diploma & $-2 \cdot 61$ & 0.36 & $<0.001$ & $-2 \cdot 61$ & 0.34 & $<0.001$ \\
\hline \multicolumn{7}{|c|}{ Index of Relative Socio-economic Disadvantage } \\
\hline 1st quintile (most disadvantaged) & 0 & Ref. & & 0 & Ref. & \\
\hline 2nd quintile & $-1 \cdot 26$ & 0.51 & 0.01 & $-1 \cdot 40$ & 0.48 & 0.004 \\
\hline 3rd quintile & -1.54 & 0.51 & 0.003 & -1.95 & 0.48 & $<0.001$ \\
\hline 4th quintile & $-2 \cdot 02$ & 0.50 & $<0.001$ & $-2 \cdot 29$ & 0.47 & $<0.001$ \\
\hline 5th quintile (least disadvantaged) & $-2 \cdot 74$ & 0.48 & $<0.001$ & $-3 \cdot 12$ & 0.45 & $<0.001$ \\
\hline \multicolumn{7}{|l|}{ Marital status } \\
\hline Married & 0 & Ref. & & 0 & Ref. & \\
\hline Single & $-0 \cdot 76$ & 0.50 & $0 \cdot 13$ & -0.60 & 0.47 & $0 \cdot 20$ \\
\hline Divorced & $-0 \cdot 19$ & 0.53 & $0 \cdot 72$ & -0.66 & 0.50 & $0 \cdot 19$ \\
\hline De facto & $0 \cdot 20$ & 0.93 & 0.83 & -0.06 & 0.87 & 0.95 \\
\hline Widowed & $-0 \cdot 38$ & 0.62 & 0.54 & -0.06 & 0.58 & 0.92 \\
\hline Separated & $-2 \cdot 55$ & 0.98 & 0.009 & -1.98 & 0.92 & 0.03 \\
\hline Height (per $10 \mathrm{~cm}$ ) & $6 \cdot 76$ & 0.23 & $<0.001$ & $1 \cdot 78$ & $0 \cdot 21$ & $<0.001$ \\
\hline Diastolic blood pressure (per $10 \mathrm{mmHg}$ ) & $1 \cdot 76$ & $0 \cdot 13$ & $<0.001$ & 1.62 & $0 \cdot 12$ & $<0.001$ \\
\hline Systolic blood pressure (per $10 \mathrm{mmHg}$ ) & $1 \cdot 24$ & 0.09 & $<0.001$ & $1 \cdot 13$ & 0.08 & $<0.001$ \\
\hline Pulse (per 10 beats $/ \mathrm{min}$ ) & 0.92 & $0 \cdot 15$ & $<0.001$ & 1.03 & $0 \cdot 14$ & $<0.001$ \\
\hline \multicolumn{7}{|l|}{ Walking } \\
\hline None & 0 & Ref. & & 0 & Ref. & \\
\hline $1-2$ times/week & -0.33 & $0 \cdot 37$ & 0.37 & -0.45 & 0.35 & $0 \cdot 19$ \\
\hline$\geq 3$ times/week & $-1 \cdot 19$ & 0.31 & $<0.001$ & $-1 \cdot 16$ & 0.29 & $<0.001$ \\
\hline \multicolumn{7}{|l|}{ Physical activity score } \\
\hline 0 & 0 & Ref. & & 0 & Ref. & \\
\hline $1-3$ & $-1 \cdot 39$ & 0.44 & 0.001 & $-1 \cdot 19$ & 0.41 & 0.004 \\
\hline $4-5$ & $-2 \cdot 20$ & $0 \cdot 40$ & $<0.001$ & -1.68 & 0.37 & $<0.001$ \\
\hline$\geq 6$ & $-3 \cdot 79$ & 0.42 & $<0.001$ & -3.84 & $0 \cdot 40$ & $<0.001$ \\
\hline \multicolumn{7}{|l|}{ Exerciset } \\
\hline None & 0 & Ref. & & 0 & Ref. & \\
\hline Less vigorous only ( $\geq$ once/week) & $-1 \cdot 34$ & 0.33 & $<0.001$ & -1.35 & 0.31 & $<0.001$ \\
\hline Vigorous ( $\geq$ once/week) & $-2 \cdot 82$ & 0.35 & $<0.001$ & $-2 \cdot 93$ & 0.33 & $<0.001$ \\
\hline \multicolumn{7}{|l|}{ Moderate/heavy activity at work } \\
\hline No & 0 & Ref. & & 0 & Ref. & \\
\hline Yes & -0.55 & 0.45 & 0.23 & $-1 \cdot 12$ & 0.42 & 0.008 \\
\hline \multicolumn{7}{|l|}{ Moderate/heavy activity at home } \\
\hline No & 0 & Ref. & & 0 & Ref. & \\
\hline Yes & -0.05 & $0 \cdot 29$ & $0 \cdot 87$ & -0.05 & $0 \cdot 27$ & $0 \cdot 84$ \\
\hline Smoking & & & & & & \\
\hline Never & 0 & Ref. & & 0 & Ref. & \\
\hline Past & 0.45 & $0 \cdot 30$ & $0 \cdot 13$ & 0.37 & 0.28 & $0 \cdot 19$ \\
\hline Total alcohol & & & & & & \\
\hline Lifetime abstainers & 0 & Ref. & & 0 & Ref. & \\
\hline $0.1-19.9 \mathrm{~g} / \mathrm{d}$ & $-1 \cdot 10$ & 0.33 & 0.001 & -0.74 & $0 \cdot 31$ & 0.02 \\
\hline $20.0-39 \cdot 9 \mathrm{~g} / \mathrm{d}$ & $-1 \cdot 60$ & 0.48 & 0.001 & -0.68 & 0.45 & $0 \cdot 13$ \\
\hline$\geq 40.0 \mathrm{~g} / \mathrm{d}$ & 0.06 & 0.62 & 0.93 & 0.77 & 0.58 & $0 \cdot 19$ \\
\hline Alcohol-specific & & & & & & \\
\hline Lifetime abstainers & 0 & Ref. & & 0 & Ref. & \\
\hline Beer & -0.38 & 0.38 & 0.31 & 0.44 & 0.35 & $0 \cdot 21$ \\
\hline Wine & $-1 \cdot 22$ & 0.32 & $<0.001$ & -0.77 & $0 \cdot 31$ & 0.01 \\
\hline Spirits & $-0 \cdot 76$ & $0 \cdot 36$ & 0.03 & -0.50 & $0 \cdot 34$ & $0 \cdot 14$ \\
\hline Energy intake $(\mathrm{MJ} / \mathrm{d})$ & 0.04 & 0.05 & 0.42 & 0.06 & 0.04 & $0 \cdot 15$ \\
\hline Protein (per $10 \%$ of energy) & $2 \cdot 87$ & 0.54 & $<0.001$ & $2 \cdot 45$ & 0.48 & $<0.001$ \\
\hline Fat (per $10 \%$ of energy) & $1 \cdot 61$ & $0 \cdot 26$ & $<0.001$ & 1.59 & 0.23 & $<0.001$ \\
\hline Carbohydrates (per $10 \%$ of energy) & -1.51 & 0.22 & $<0.001$ & -1.58 & $0 \cdot 19$ & $<0.001$ \\
\hline Fibre (per $1 \mathrm{MJ}$ of energy) & -0.52 & $0 \cdot 16$ & 0.001 & -0.81 & $0 \cdot 14$ & $<0.001$ \\
\hline
\end{tabular}

$\beta$, coefficient; Ref., referent category.

${ }^{*}$ Adjusted for sex, height, age, highest level of education (pseudo-continuous), physical activity score (pseudo-continuous), marital status (married/de facto $v$. single/divorced/widowed/separated), Index of Relative Socio-economic Disadvantage (pseudo-continuous), smoking status and dietary energy intake at baseline attendance.

tParticipants in the vigorous exercise group may have also reported doing less vigorous exercise.

‡Participants in the alcohol-specific groups may have also reported drinking other types of alcoholic beverages. 
Table 3 Associations of potential predictors of weight and waist circumference at wave 2: adults with data at baseline (1990-1994) and wave 2 (2003-2007), Melbourne Collaborative Cohort Study

\begin{tabular}{|c|c|c|c|c|c|c|}
\hline & \multicolumn{3}{|c|}{ Weight $(\mathrm{kg})$ at wave $2^{*}$} & \multicolumn{3}{|c|}{ Waist circumference $(\mathrm{cm})$ at wave $2 \dagger$} \\
\hline & $\beta$ & SE & $P$ value & $\beta$ & SE & $P$ value \\
\hline Baseline weight (per 10 kg) & $10 \cdot 64$ & 0.06 & $<0.001$ & $\mathrm{~N} / \mathrm{A}$ & & \\
\hline Baseline waist (per $10 \mathrm{~cm}$ ) & $\mathrm{N} / \mathrm{A}$ & & & $8 \cdot 90$ & $0 \cdot 10$ & $<0.001$ \\
\hline \multicolumn{7}{|l|}{ Sex } \\
\hline Male & 0 & Ref. & & 0 & Ref. & \\
\hline Female & $1 \cdot 38$ & $0 \cdot 20$ & $<0.001$ & $1 \cdot 25$ & 0.31 & $<0.001$ \\
\hline Age at baseline attendance (per 10 years) & $-1 \cdot 75$ & 0.08 & $<0.001$ & -0.58 & $0 \cdot 12$ & $<0.001$ \\
\hline \multicolumn{7}{|l|}{ Country of birth } \\
\hline Australia/New Zealand/UK & 0 & Ref. & & 0 & Ref. & \\
\hline Greece/ltaly & 0.04 & $0 \cdot 10$ & 0.69 & 0.39 & $0 \cdot 14$ & 0.006 \\
\hline Follow-up time (per year) & 0.46 & 0.05 & $<0.001$ & 0.80 & 0.08 & $<0.001$ \\
\hline \multicolumn{7}{|l|}{ Education level } \\
\hline Primary, some high/technical school & 0 & Ref. & & 0 & Ref. & \\
\hline Completed high school & 0.03 & $0 \cdot 18$ & $0 \cdot 88$ & -0.42 & $0 \cdot 26$ & $0 \cdot 10$ \\
\hline Completed tertiary degree/diploma & -0.27 & $0 \cdot 18$ & $0 \cdot 13$ & -1.07 & $0 \cdot 25$ & $<0.001$ \\
\hline \multicolumn{7}{|l|}{ Index of Relative Socio-economic Disadvantage } \\
\hline 1st quintile (most disadvantaged) & 0 & Ref. & & 0 & Ref. & \\
\hline 2nd quintile & $0 \cdot 18$ & 0.25 & 0.48 & 0.32 & 0.36 & 0.38 \\
\hline 3rd quintile & -0.35 & 0.25 & $0 \cdot 16$ & -0.54 & 0.36 & $0 \cdot 13$ \\
\hline 4th quintile & -0.47 & 0.24 & 0.05 & -0.83 & 0.35 & 0.02 \\
\hline 5th quintile (least disadvantaged) & $-0 \cdot 72$ & 0.24 & 0.002 & $-1 \cdot 16$ & 0.34 & 0.001 \\
\hline \multicolumn{7}{|l|}{ Marital status } \\
\hline Married & 0 & Ref. & & 0 & Ref. & \\
\hline Single & 0.55 & 0.24 & 0.02 & 0.51 & 0.35 & $0 \cdot 14$ \\
\hline Divorced & $1 \cdot 04$ & 0.26 & $<0.001$ & 1.59 & 0.37 & $<0.001$ \\
\hline De facto & $-0 \cdot 18$ & 0.45 & $0 \cdot 70$ & 0.02 & 0.64 & 0.97 \\
\hline Widowed & $1 \cdot 01$ & $0 \cdot 30$ & 0.001 & $0 \cdot 20$ & 0.43 & 0.64 \\
\hline Separated & 0.61 & 0.48 & 0.20 & $0 \cdot 17$ & 0.68 & $0 \cdot 80$ \\
\hline Height (per $10 \mathrm{~cm})$ & -0.08 & $0 \cdot 12$ & 0.49 & 0.39 & $0 \cdot 16$ & 0.01 \\
\hline Diastolic blood pressure (per $10 \mathrm{mmHg}$ ) & -0.03 & 0.07 & 0.68 & 0.39 & 0.09 & $<0.001$ \\
\hline Systolic blood pressure (per $10 \mathrm{mmHg}$ ) & -0.03 & 0.04 & 0.55 & 0.24 & 0.06 & $<0.001$ \\
\hline Pulse (per 10 beats/min) & -0.06 & 0.07 & 0.39 & 0.35 & $0 \cdot 10$ & 0.001 \\
\hline \multicolumn{7}{|l|}{ Walking } \\
\hline None & 0 & Ref. & & 0 & Ref. & \\
\hline 1-2 times/week & 0.02 & $0 \cdot 18$ & 0.93 & -0.07 & $0 \cdot 25$ & 0.77 \\
\hline$\geq 3$ times/week & -0.03 & $0 \cdot 15$ & 0.85 & -0.23 & $0 \cdot 22$ & $0 \cdot 28$ \\
\hline \multicolumn{7}{|l|}{ Physical activity score } \\
\hline 0 & 0 & Ref. & & 0 & Ref. & \\
\hline $1-3$ & -0.24 & $0 \cdot 21$ & $0 \cdot 26$ & -0.47 & $0 \cdot 30$ & $0 \cdot 12$ \\
\hline $4-5$ & -0.25 & 0.19 & $0 \cdot 20$ & -0.73 & $0 \cdot 28$ & 0.008 \\
\hline$\geq 6$ & -0.43 & $0 \cdot 21$ & 0.04 & $-1 \cdot 20$ & $0 \cdot 30$ & $<0.001$ \\
\hline \multicolumn{7}{|l|}{ Exerciseł } \\
\hline None & 0 & Ref. & & 0 & Ref. & \\
\hline Less vigorous only ( $\geq$ once/week) & $-0 \cdot 18$ & $0 \cdot 16$ & $0 \cdot 27$ & -0.53 & 0.23 & 0.02 \\
\hline Vigorous ( $\geq$ once/week) & -0.46 & $0 \cdot 17$ & 0.006 & $-1 \cdot 11$ & $0 \cdot 24$ & $<0.001$ \\
\hline \multicolumn{7}{|l|}{ Moderate/heavy activity at work } \\
\hline No & 0 & Ref. & & 0 & Ref. & \\
\hline Yes & 0.39 & 0.22 & 0.08 & 0.84 & 0.31 & 0.007 \\
\hline Moderate/heavy activity at home & & & & & & \\
\hline No & 0 & Ref. & & 0 & Ref. & \\
\hline Yes & 0.03 & 0.14 & 0.83 & -0.07 & $0 \cdot 20$ & 0.73 \\
\hline Smoking & & & & & & \\
\hline Never & 0 & Ref. & & 0 & Ref. & \\
\hline Past & -0.06 & $0 \cdot 15$ & $0 \cdot 70$ & 0.26 & $0 \cdot 21$ & 0.20 \\
\hline Total alcohol & & & & & & \\
\hline Lifetime abstainers & 0 & Ref. & & 0 & Ref. & \\
\hline $0 \cdot 1-19 \cdot 9 \mathrm{~g} / \mathrm{d}$ & -0.23 & $0 \cdot 16$ & $0 \cdot 15$ & -0.58 & $0 \cdot 23$ & 0.01 \\
\hline $20.0-39.9 \mathrm{~g} / \mathrm{d}$ & -0.48 & 0.23 & 0.04 & -1.07 & 0.33 & 0.001 \\
\hline$\geq 40.0 \mathrm{~g} / \mathrm{d}$ & -0.27 & 0.30 & 0.37 & -0.13 & 0.43 & $0 \cdot 76$ \\
\hline Alcohol-specific $\$$ & & & & & & \\
\hline Lifetime abstainers & 0 & Ref. & & 0 & Ref. & \\
\hline Beer & -0.41 & $0 \cdot 18$ & 0.03 & -0.82 & $0 \cdot 26$ & 0.002 \\
\hline Wine & -0.25 & $0 \cdot 16$ & $0 \cdot 12$ & -0.67 & 0.23 & 0.003 \\
\hline Spirits & -0.37 & $0 \cdot 17$ & 0.03 & -0.59 & 0.25 & 0.02 \\
\hline Energy intake $(\mathrm{MJ} / \mathrm{d})$ & 0.00 & 0.02 & 0.85 & -0.03 & 0.03 & $0 \cdot 31$ \\
\hline Protein (per $10 \%$ of energy) & $0 \cdot 81$ & 0.25 & 0.001 & $1 \cdot 85$ & 0.35 & $<0.001$ \\
\hline Fat (per $10 \%$ of energy) & 0.26 & $0 \cdot 12$ & 0.03 & 0.85 & $0 \cdot 17$ & $<0.001$ \\
\hline Carbohydrates (per $10 \%$ of energy) & -0.19 & $0 \cdot 10$ & 0.06 & -0.73 & $0 \cdot 14$ & $<0.001$ \\
\hline Fibre (per $1 \mathrm{MJ}$ of energy) & -0.07 & 0.07 & 0.36 & -0.27 & $0 \cdot 10$ & 0.009 \\
\hline
\end{tabular}

$\beta$, coefficient; N/A, not applicable; Ref., referent category.

${ }^{*}$ Adjusted for weight, sex, height, age, highest level of education (pseudo-continuous), physical activity score (pseudo-continuous), marital status (married/de facto $v$. single/divorced/widowed/separated), Index of Relative Socio-economic Disadvantage (pseudo-continuous), smoking status and dietary energy intake at baseline attendance.

tAdjusted for waist circumference, sex, height, age, highest level of education (pseudo-continuous), physical activity score (pseudo-continuous), marital status (married/de facto $v$. single/divorced/widowed/separated), Index of Relative Socio-economic Disadvantage (pseudo-continuous), smoking status and dietary energy intake at baseline attendance.

‡Participants in the vigorous exercise group may have also reported doing less vigorous exercise.

$\S$ Participants in the alcohol-specific groups may have also reported drinking other types of alcoholic beverages. 
Table 4 Selected associations of potential predictors of weight at wave 2 by age at attendance*: adults with data at baseline (1990-1994) and wave 2 (2003-2007), Melbourne Collaborative Cohort Study

\begin{tabular}{|c|c|c|c|c|c|c|c|c|c|c|}
\hline & \multicolumn{3}{|c|}{ 40-49 years } & \multicolumn{3}{|c|}{ 50-59 years } & \multicolumn{3}{|c|}{ 60-69 years } & \multirow[b]{2}{*}{$P$ for interaction } \\
\hline & $\beta$ & SE & $P$ value & $\beta$ & SE & $P$ value & $\beta$ & SE & $P$ value & \\
\hline Walking & & & & & & & & & & 0.38 \\
\hline None & 0 & Ref. & & 0 & Ref. & & 0 & Ref. & & \\
\hline $1-2$ times/week & 0.07 & 0.31 & 0.83 & $0 \cdot 18$ & $0 \cdot 31$ & 0.56 & -0.34 & 0.29 & 0.24 & \\
\hline$\geq 3$ times/week & 0.00 & 0.27 & 1.00 & $0 \cdot 14$ & $0 \cdot 26$ & 0.59 & -0.41 & 0.23 & 0.08 & \\
\hline Physical activity score & & & & & & & & & & 0.25 \\
\hline 0 & 0 & Ref. & & 0 & Ref. & & 0 & Ref. & & \\
\hline $1-3$ & -0.51 & 0.38 & $0 \cdot 19$ & $0 \cdot 16$ & 0.35 & 0.66 & -0.39 & 0.34 & 0.25 & \\
\hline $4-5$ & -0.51 & $0 \cdot 36$ & $0 \cdot 16$ & $0 \cdot 16$ & 0.32 & 0.61 & -0.44 & 0.30 & $0 \cdot 15$ & \\
\hline$\geq 6$ & -0.77 & 0.36 & 0.03 & -0.05 & 0.36 & 0.88 & -0.32 & 0.35 & 0.35 & \\
\hline \multicolumn{11}{|l|}{ Exerciset } \\
\hline None & 0 & Ref. & & 0 & Ref. & & 0 & Ref. & & \\
\hline Less vigorous only ( $\geq$ once/week) & -0.83 & 0.32 & 0.01 & 0.02 & $0 \cdot 27$ & 0.95 & 0.24 & 0.23 & 0.31 & 0.009 \\
\hline Vigorous ( $\geq$ once/week) & -0.89 & 0.28 & 0.001 & -0.32 & 0.30 & 0.28 & 0.30 & 0.30 & 0.33 & 0.001 \\
\hline \multicolumn{11}{|l|}{ Moderate/heavy activity at work } \\
\hline No & 0 & Ref. & & 0 & Ref. & & 0 & Ref. & & \\
\hline Yes & 0.51 & 0.36 & $0 \cdot 15$ & 0.39 & 0.35 & 0.27 & $-0 \cdot 10$ & 0.51 & 0.85 & 0.23 \\
\hline \multicolumn{11}{|l|}{ Moderate/heavy activity at home } \\
\hline No & 0 & Ref. & & 0 & Ref. & & 0 & Ref. & & \\
\hline Yes & -0.01 & 0.26 & 0.98 & 0.36 & 0.24 & $0 \cdot 14$ & -0.31 & $0 \cdot 21$ & $0 \cdot 14$ & 0.46 \\
\hline Total alcohol & & & & & & & & & & $0 \cdot 19$ \\
\hline Lifetime abstainers & 0 & Ref. & & 0 & Ref. & & 0 & Ref. & & \\
\hline $0.1-19.9 \mathrm{~g} / \mathrm{d}$ & -0.31 & 0.31 & 0.31 & -0.03 & 0.27 & 0.91 & -0.35 & $0 \cdot 24$ & $0 \cdot 14$ & \\
\hline $20.0-39.9 \mathrm{~g} / \mathrm{d}$ & -0.74 & 0.43 & 0.08 & -0.61 & 0.41 & $0 \cdot 13$ & 0.04 & 0.36 & 0.91 & \\
\hline$\geq 40.0 \mathrm{~g} / \mathrm{d}$ & -0.65 & 0.57 & 0.26 & 0.22 & 0.50 & 0.66 & -0.35 & 0.46 & 0.45 & \\
\hline Energy intake $(\mathrm{MJ} / \mathrm{d})$ & 0.02 & 0.04 & 0.67 & 0.00 & 0.04 & 0.92 & -0.06 & 0.04 & 0.09 & 0.71 \\
\hline Protein (per $10 \%$ of energy) & $0 \cdot 77$ & 0.47 & $0 \cdot 10$ & 0.69 & $0 \cdot 42$ & $0 \cdot 10$ & 0.99 & 0.37 & 0.008 & 0.44 \\
\hline Fat (per $10 \%$ of energy) & $0 \cdot 70$ & 0.22 & 0.002 & -0.01 & 0.20 & 0.94 & 0.05 & $0 \cdot 18$ & 0.79 & 0.001 \\
\hline Carbohydrates (per $10 \%$ of energy) & -0.32 & $0 \cdot 19$ & 0.08 & -0.09 & $0 \cdot 17$ & 0.60 & $-0 \cdot 17$ & $0 \cdot 16$ & 0.28 & $0 \cdot 15$ \\
\hline Fibre (per $1 \mathrm{MJ}$ of energy) & -0.29 & $0 \cdot 13$ & 0.03 & 0.05 & $0 \cdot 12$ & 0.72 & $0 \cdot 14$ & $0 \cdot 12$ & 0.23 & 0.02 \\
\hline
\end{tabular}

$\beta$, coefficient; Ref., referent category.

*Adjusted for weight, sex, height, age, highest level of education (pseudo-continuous), physical activity score (pseudo-continuous), marital status (married/de facto $v$. single/divorced/widowed/separated), Index of Relative Socio-economic Disadvantage (pseudo-continuous), smoking status and dietary energy intake at baseline attendance.

tParticipants in the vigorous exercise group may have also reported doing less vigorous exercise.

Analyses stratified by sex indicated that male former smokers were more likely to have a greater waist circumference at wave 2 (males $\beta$ coefficient $=0 \cdot 87, \mathrm{sE}=0 \cdot 28$, $P=0 \cdot 002$; females $\beta$ coefficient $=0 \cdot 13$, se $=0 \cdot 29, P=0 \cdot 67$; $P$ for interaction $=0 \cdot 02$ ). Compared with abstainers, female heavy drinkers of alcohol $(\geq 40 \cdot 0 \mathrm{~g} / \mathrm{d})$ were more likely to have a smaller waist circumference at wave 2 (males $\beta$ coefficient $=0.62$, SE $=0.51, P=0 \cdot 23$; females $\beta$ coefficient $=-2 \cdot 00, \mathrm{se}=0 \cdot 86, P=0 \cdot 02 ; P$ for interaction $=$ $0 \cdot 003)$. There were no other notable differences in results between males and females (data not shown).

\section{Discussion}

We have shown that some of the factors that predict greater weight and larger waist circumference in middle-aged and older adults over a 12-year follow-up period, such as physical activity and diet, are modifiable targets for individual behaviour change. Similar interventions could successfully address the management of weight and waist circumference, as both measures are predicted by similar factors.

One strength of our study is that weight and waist circumference were directly measured on both occasions, thus minimizing potential errors that can occur with selfreported measures. Restriction of the analyses to people who were not current smokers and did not report having been diagnosed with cancer, diabetes, angina, stroke or heart disease at baseline reduced the likelihood that the results are explained by confounding due to smoking or to reverse causation due to prevalent illness ${ }^{(12)}$. It is possible that some participants may not have known they had diabetes, but it is unlikely that they would have changed their reported lifestyle habits at baseline because of this. On the other hand, participants who were aware they had diabetes at baseline may have altered their lifestyle in response to the diagnosis. While some studies have analysed predictors of weight change, we analysed absolute weight at wave 2 conditional on weight at baseline, as there is considerable doubt that causal knowledge may be gained by substituting a derived variable (i.e. change in weight) for a measured variable $^{(21)}$. In addition, our approach does not restrict the relationship between values at baseline and wave 2 in the way that analysing change does.

The major limitation is the loss to follow-up of 2571 eligible people, of whom 1894 declined to attend for physical examination at wave 2 . Because they differed in 
Table 5 Selected associations of potential predictors of waist circumference at wave 2 by age at attendance*: adults with data at baseline (1990-1994) and wave 2 (2003-2007), Melbourne Collaborative Cohort Study

\begin{tabular}{|c|c|c|c|c|c|c|c|c|c|c|}
\hline & \multicolumn{3}{|c|}{$40-49$ years } & \multicolumn{3}{|c|}{$50-59$ years } & \multicolumn{3}{|c|}{$60-69$ years } & \multirow[b]{2}{*}{$P$ for interaction } \\
\hline & $\beta$ & SE & $P$ value & $\beta$ & SE & $P$ value & $\beta$ & SE & $P$ value & \\
\hline Walking & & & & & & & & & & $0 \cdot 15$ \\
\hline None & 0 & Ref. & & 0 & Ref. & & 0 & Ref. & & \\
\hline 1-2 times per week & $-0 \cdot 10$ & 0.43 & $0 \cdot 82$ & 0.01 & 0.42 & 0.98 & -0.23 & 0.47 & 0.63 & \\
\hline$\geq 3$ times per week & -0.05 & 0.38 & 0.90 & -0.01 & $0 \cdot 35$ & 0.97 & $-0 \cdot 80$ & 0.38 & 0.04 & \\
\hline Physical activity score & & & & & & & & & & $0 \cdot 71$ \\
\hline 0 & 0 & Ref. & & 0 & Ref. & & 0 & Ref. & & \\
\hline $1-3$ & -1.04 & 0.53 & 0.05 & $0 \cdot 17$ & 0.48 & $0 \cdot 72$ & -0.60 & 0.55 & $0 \cdot 28$ & \\
\hline $4-5$ & $-1 \cdot 16$ & 0.49 & 0.02 & -0.02 & 0.43 & $0 \cdot 96$ & $-1 \cdot 01$ & 0.49 & 0.04 & \\
\hline$\geq 6$ & $-1 \cdot 48$ & 0.49 & 0.003 & -0.58 & 0.49 & $0 \cdot 24$ & $-1 \cdot 50$ & 0.56 & 0.007 & \\
\hline \multicolumn{11}{|l|}{ Exerciset } \\
\hline None & 0 & Ref. & & 0 & Ref. & & 0 & Ref. & & \\
\hline Less vigorous only ( $\geq$ once/week) & $-1 \cdot 42$ & 0.45 & 0.002 & 0.05 & $0 \cdot 37$ & 0.90 & -0.22 & 0.38 & 0.55 & $0 \cdot 11$ \\
\hline Vigorous ( $\geq$ once/week) & $-1 \cdot 67$ & 0.39 & $<0.001$ & -0.79 & $0 \cdot 41$ & 0.05 & -0.35 & 0.50 & 0.49 & 0.01 \\
\hline \multicolumn{11}{|l|}{ Moderate/heavy activity at work } \\
\hline No & 0 & Ref. & & 0 & Ref. & & 0 & Ref. & & \\
\hline Yes & 1.00 & 0.50 & 0.04 & $1 \cdot 12$ & $0 \cdot 47$ & 0.02 & $-0 \cdot 87$ & $0 \cdot 82$ & $0 \cdot 29$ & $0 \cdot 10$ \\
\hline \multicolumn{11}{|l|}{ Moderate/heavy activity at home } \\
\hline No & 0 & Ref. & & 0 & Ref. & & 0 & Ref. & & \\
\hline Yes & $-0 \cdot 27$ & $0 \cdot 36$ & 0.45 & 0.57 & $0 \cdot 32$ & 0.08 & -0.63 & 0.34 & 0.07 & 0.69 \\
\hline Total alcohol & & & & & & & & & & 0.54 \\
\hline Lifetime abstainers & 0 & Ref. & & 0 & Ref. & & 0 & Ref. & & \\
\hline $0 \cdot 1-19 \cdot 9 \mathrm{~g} / \mathrm{d}$ & -0.78 & $0 \cdot 42$ & 0.07 & -0.40 & $0 \cdot 37$ & $0 \cdot 27$ & -0.46 & 0.39 & $0 \cdot 24$ & \\
\hline $20.0-39.9 \mathrm{~g} / \mathrm{d}$ & -0.96 & 0.59 & $0 \cdot 11$ & $-1 \cdot 29$ & 0.55 & 0.02 & -0.99 & 0.58 & 0.09 & \\
\hline$\geq 40.0 \mathrm{~g} / \mathrm{d}$ & 0.45 & $0 \cdot 79$ & 0.56 & -0.20 & 0.69 & $0 \cdot 78$ & -0.62 & 0.75 & $0 \cdot 41$ & \\
\hline Energy intake $(\mathrm{MJ} / \mathrm{d})$ & -0.02 & 0.06 & 0.79 & -0.05 & 0.05 & $0 \cdot 36$ & -0.04 & 0.06 & 0.48 & 0.46 \\
\hline Protein (per $10 \%$ of energy) & $2 \cdot 38$ & 0.65 & $<0.001$ & $1 \cdot 21$ & 0.56 & 0.03 & $1 \cdot 81$ & 0.60 & 0.003 & 0.09 \\
\hline Fat (per $10 \%$ of energy) & $1 \cdot 32$ & $0 \cdot 31$ & $<0.001$ & 0.68 & $0 \cdot 28$ & 0.01 & 0.43 & $0 \cdot 29$ & $0 \cdot 14$ & 0.02 \\
\hline Carbohydrates (per $10 \%$ of energy) & $-1 \cdot 21$ & $0 \cdot 26$ & $<0.001$ & -0.51 & $0 \cdot 23$ & 0.03 & -0.36 & 0.25 & $0 \cdot 16$ & 0.02 \\
\hline Fibre (per $1 \mathrm{MJ}$ of energy) & -0.58 & $0 \cdot 18$ & 0.001 & $-0 \cdot 15$ & $0 \cdot 17$ & 0.38 & 0.00 & $0 \cdot 19$ & 0.98 & 0.08 \\
\hline
\end{tabular}

$\beta$, coefficient; Ref., referent category.

${ }^{*}$ Adjusted for waist circumference, sex, height, age, highest level of education (pseudo-continuous), physical activity score (pseudo-continuous), marital status (married/de facto $v$. single/divorced/widowed/separated), Index of Relative Socio-economic Disadvantage (pseudo-continuous), smoking status and dietary energy intake at baseline attendance.

tParticipants in the vigorous exercise group may have also reported doing less vigorous exercise.

their baseline characteristics from those retained, selection bias is a possibility. We were unable to find any association with energy intake, which might be because FFQ are not good at measuring absolute energy. They are better at measuring composition and patterns of the diet (i.e. how much the diet is comprised of protein, fat, etc. ${ }^{(22)}$, which might explain our findings in this regard. It should also be noted that the questions asked on physical activity pertained to frequency, but not duration. We had no information on sedentary behaviour or sleep duration, which are also associated with weight gain ${ }^{(1,3)}$. We had measured behaviours at baseline, and if these changed markedly over the 12 years between waves, attenuation of associations is possible. Regression to the mean was unlikely to have had a major impact on results, since body weight and waist circumference are not highly prone to diurnal variability or reader or other measurement error ${ }^{(23)}$.

Like us, Ellaway and colleagues found that neighbourhood of residence was associated with waist circumference after controlling for individual measures of affluence $^{(24)}$, and living in a socio-economically deprived area is consistently associated with higher weight ${ }^{(3,25)}$.
These findings suggest that targeting individual behaviour alone is unlikely to reduce the proportion of overweight individuals in the population. Changing obesity-promoting environments to increase physical activity, reduce sedentary behaviour, increase the availability of healthier food products and decrease the availability of unhealthy food products should impact on a large proportion of the population and support individual behaviour change ${ }^{(26)}$. While difficult and expensive, such changes might be achieved through public policy, transport infrastructure, urban design and taxation. Any public education campaigns to improve eating habits and levels of physical activity should target disadvantaged population groups.

We found that people who were married or in de facto arrangements had lower weight and waist circumference at wave 2 than people who reported being single (widowed, divorced, separated or single), suggesting the importance of intimate social relationships to weight maintenance. On the other hand, a recent publication from the Whitehall II Study showed that adverse social relationships may contribute to weight and waist circumference increases over a 12 -year period ${ }^{(27)}$. While we did not evaluate the quality of relationships, it may be that 
people living with partners had generally better social relationships than those on their own, or that being single was a marker of adverse social relationships. Future studies could explore how the number and quality of social relationships of various levels of intimacy relate to weight gain.

Waist circumference (but not weight) at wave 2 was positively associated with blood pressure at baseline. Elevated blood pressure is part of the constellation of abnormalities constituting the metabolic syndrome, along with abdominal obesity, dyslipidaemia, dysglycaemia and insulin resistance ${ }^{(28)}$, and it may be that behavioural and genetic factors contributing to rising blood pressure also contribute to visceral fat accumulation. Adjusting for baseline waist circumference may not fully account for visceral adiposity as the external waist measurement also includes subcutaneous fat.

In 2007, the World Cancer Research Fund and American Institute for Cancer Research panel concluded there was convincing evidence that physical activity of all types protects against weight gain ${ }^{(3)}$. Conversely, a more recent systematic review by Summerbell and colleagues concluded that the evidence was weak and inconsistent ${ }^{(2)}$. As there is much heterogeneity between different study designs (including age, sex and baseline weight of participants, duration of follow-up, measurement of the exposure, inclusion and exclusion of participants who smoke or have a pre-existing chronic disease), it is difficult to draw meaningful conclusions. The evidence for an association with leisure-time physical activity, however, is more convincing. Two of three intervention trials in adults (see detailed review in reference 3 ) showed that weight decreased with exercise aimed at weight-loss maintenance. Several epidemiological studies have also shown some benefit of leisure-time physical activity on maintenance of weight and waist circumference ${ }^{(1,3,29)}$. Two ${ }^{(30,31)}$ of the three cohort studies ${ }^{(30-32)}$ that reported results for high-intensity physical activity showed inverse associations with future weight.

Previous studies have reported mixed associations between work-related physical activity and weight change (see detailed review in reference 3). These associations are likely to differ by SES. We found a positive association between moderate to heavy activity at work and future waist circumference, but not weight, even after adjusting for area-based SES, education level and leisuretime exercise. This finding, though, could be explained in part by participants no longer performing work-related activities by wave 2 due to retirement from work.

Limited information is available for the relationship between physical activity at home and weight or waist change. One study reported no association for women ${ }^{(6)}$, but another showed a slightly reduced risk of becoming obese for women who spent $40 \mathrm{~h}$ or more per week standing or walking around at home compared with inactive women ${ }^{(33)}$. We did not observe any associations for this variable and it was likely that this would have been more difficult to recall accurately than leisure-time activity, especially vigorous activity.

Several cohort studies have reported inconsistent findings with regard to alcohol intake and weight gain (see detailed review in Summerbell et al. ${ }^{(2)}$ ). Similarly, the evidence for an association between alcohol intake and change in waist circumference is not convincing ${ }^{(7,34,35)}$. Our results showed that low to moderate alcohol consumption was inversely associated with future weight and waist circumference, even after adjustment for various potential confounders.

The lack of association between total energy intake and future weight or waist circumference for our population is consistent with results from most other epidemiological studies (reviewed in Summerbell et $a l^{(2)}$ ). Obesity is determined by the imbalance of energy intake and energy expenditure $^{(36)}$, but it is difficult to differentiate small differences in energy intake (assuming energy expenditure is constant) using FFQ. Additionally, under-reporting food intake is more common in heavier people ${ }^{(37)}$. The ability to predict future weight and waist circumference based on total energy intake is also limited as people may change their dietary intakes over time.

In most published studies, carbohydrate as a percentage of energy intake at baseline was not associated with change in weight or waist circumference ${ }^{(2)}$, although some studies (including ours) report negative associations with weight gain ${ }^{(38,39)}$. Results from a meta-regression showed that fat intake as a percentage of total energy intake was not associated with change in weight (effect size $=0 \cdot 07,95 \% \mathrm{CI}-0 \cdot 03,0 \cdot 16)^{(2)}$. However, a couple of studies not included in the meta-analysis reported positive associations between fat intake and weight gain ${ }^{(32,40)}$. Protein intake (typically assessed from an FFQ) was not associated with weight gain in most previous studies ${ }^{(2)}$, but results were inconsistent. There is also evidence that protein is associated with satiety and could help with weight $\operatorname{loss}^{(41)}$. In contrast, two studies showed that protein intake was inversely associated with changes in waist circumference ${ }^{(39,42)}$. Further research is needed to evaluate how subdivisions of energy, protein, fat and carbohydrate intakes are associated with weight gain.

Findings from the current prospective cohort study indicate that promoting vigorous exercise and encouraging a high-carbohydrate, low-fat and low-protein diet could be promising approaches for obesity prevention in middle age. These recommendations accord with current behavioural recommendations for chronic disease prevention (e.g. Dietary Guidelines for Australian Adults ${ }^{(43)}$, National Physical Activity Guidelines for Adults ${ }^{(44)}$ ). In the present study, low to moderate alcohol consumption was linked to lower weight than was abstaining from drinking. Current Australian guidelines for alcohol use recommend people limit alcohol intake if they choose to drink (average of no more than two standard drinks per day) ${ }^{(45)}$, because for some health outcomes, alcohol 
abstinence may be beneficial. Observed associations between residing in socially disadvantaged suburbs and increased weight and waist circumference highlight the importance of modifying settings to support healthy individual behaviour. Driving environmental change and supporting low-income communities to improve their levels of physical activity and healthy eating are appropriately listed as key action areas for obesity prevention in Australia $^{(26)}$.

\section{Acknowledgements}

Sources of funding: This work was supported by infrastructure from the Cancer Council Victoria and grants from VicHealth 2001-0447 and the National Health and Medical Research Council 209057 and 251533. Conflicts of interest: The authors declare no conflict of interest. Authors' contributions: conception and design, G.G.G., D.R.E., A.M.H. and H.G.D.; analysis and interpretation of data, R.J.M., A.M.H., H.G.D., A.P., L.E.A.J., D.R.E. and G.G.G.; drafting the article, R.J.M.; revising the article critically for important intellectual content, A.M.H., H.G.D., A.P., L.E.A.J., D.R.E. and G.G.G.; final approval of the version to be published, R.J.M., A.M.H., H.G.D., A.P., L.E.A.J., D.R.E. and G.G.G. Acknowledgements: This study was made possible by the contribution of many people, including the original investigators and the diligent team who recruited the participants and who continue working on follow-up. The authors would also like to express their gratitude to the many thousands of Melbourne residents who continue to participate in the study.

\section{References}

1. Mozaffarian D, Hao T, Rimm EB et al. (2011) Changes in diet and lifestyle and long-term weight gain in women and men. N Engl J Med 364, 2392-2404.

2. Summerbell CD, Douthwaite W, Whittaker V et al. (2009) The association between diet and physical activity and subsequent excess weight gain and obesity assessed at 5 years of age or older: a systematic review of the epidemiological evidence. Int J Obes (Lond) 33, Suppl. 3, S1-S92.

3. World Cancer Research Fund \& American Institute for Cancer Research (2007) Food, Nutrition, Physical Activity and the Prevention of Cancer: A Global Perspective. Washington, DC: American Institute for Cancer Research.

4. Du H, van der A DL, Ginder V et al. (2009) Dietary energy density in relation to subsequent changes of weight and waist circumference in European men and women. PLOS One 4, e5339.

5. Romaguera D, Angquist L, Du H et al. (2011) Food composition of the diet in relation to changes in waist circumference adjusted for body mass index. PLoS One 6, e23384.

6. Sternfeld B, Wang H, Quesenberry CP Jr et al. (2004) Physical activity and changes in weight and waist circumference in midlife women: findings from the Study of Women's Health Across the Nation. Am J Epidemiol 160, 912-922.

7. Walls HL, Magliano DJ, McNeil JJ et al. (2011) Predictors of increasing waist circumference in an Australian population. Public Health Nutr 14, 870-881.
8. Rankinen T, Kim SY, Perusse L et al. (1999) The prediction of abdominal visceral fat level from body composition and anthropometry: ROC analysis. Int J Obes Relat Metab Disord 23, 801-809.

9. Elobeid MA, Padilla MA, Brock DW et al. (2010) Endocrine disruptors and obesity: an examination of selected persistent organic pollutants in the NHANES 1999-2002 data. Int J Environ Res Public Health 7, 2988-3005.

10. Fogelholm M, Anderssen S, Gunnarsdottir I et al. (2012) Dietary macronutrients and food consumption as determinants of long-term weight change in adult populations: a systematic literature review. Food Nutr Res 56, doi:10.3402/ fnr.v56i0.19103.

11. Simonsen MK, Hundrup YA, Gronbaek M et al. (2008) A prospective study of the association between weight changes and self-rated health. BMC Womens Health 8, 13.

12. Manson JE, Bassuk SS, Hu FB et al. (2007) Estimating the number of deaths due to obesity: can the divergent findings be reconciled? J Womens Health (Larchmt) 16, 168-176.

13. Giles GG \& English DR (2002) The Melbourne Collaborative Cohort Study. IARC Sci Publ 156, 69-70.

14. Lohman TG, Roche AF \& Martorell R (editors) (1988) Anthropometric Standardization Reference Manual. Champaign, IL: Human Kinetics Books.

15. MacInnis RJ, English DR, Gertig DM et al. (2003) Body size and composition and prostate cancer risk. Cancer Epidemiol Biomarkers Prev 12, 1417-1421.

16. Australian Bureau of Statistics (1996) Index of Relative Socio-economic Advantage and Disadvantage. ABS Catalogue no. 2033.0.55.001. Canberra: Commonwealth of Australia.

17. National Heart Foundation of Australia \& Australian Institute of Health (1991) Risk Factor Prevalence Study: Survey No. 3 1989, Cities Analysis. Canberra: National Heart Foundation of Australia and Australian Institute of Health.

18. Ireland P, Jolley D, Giles G et al. (1994) Development of the Melbourne FFQ: a food frequency questionnaire for use in an Australian prospective study involving an ethnically diverse cohort. Asia Pac J Clin Nutr 3, 19-31.

19. Hodge A, Patterson AJ, Brown WJ et al. (2000) The Anti Cancer Council of Victoria FFQ: relative validity of nutrient intakes compared with weighed food records in young to middle-aged women in a study of iron supplementation. Aust N Z J Public Health 24, 576-583.

20. Holland B, Welch AA, Unwin ID et al. (editors) (1991) McCance and Widdowson's The Composition of Foods, 5th ed. Cambridge: The Royal Society of Chemistry and Ministry of Agriculture, Fisheries and Food.

21. Shahar E \& Shahar DJ (2012) Causal diagrams and change variables. J Eval Clin Pract 18, 143-148.

22. Willett W (1998) Nutritional Epidemiology, 2nd ed. New York: Oxford University Press.

23. Ball K, Crawford D, Ireland $\mathrm{P}$ et al. (2003) Patterns and demographic predictors of 5-year weight change in a multi-ethnic cohort of men and women in Australia. Public Health Nutr 6, 269-281.

24. Ellaway A, Anderson A \& Macintyre S (1997) Does area of residence affect body size and shape? Int J Obes Relat Metab Disord 21, 304-308.

25. Giskes K, van Lenthe F, Avendano-Pabon M et al. (2011) A systematic review of environmental factors and obesogenic dietary intakes among adults: are we getting closer to understanding obesogenic environments? Obes Rev 12, e95-e106.

26. National Preventative Health Taskforce (2009) Obesity in Australia: A Need for Urgent Action. Canberra: Commonwealth of Australia.

27. Kouvonen A, Stafford M, De Vogli R et al. (2011) Negative aspects of close relationships as a predictor of increased body mass index and waist circumference: the Whitehall II study. Am J Public Health 101, 1474-1480. 
28. Despres JP, Lemieux I, Bergeron J et al. (2008) Abdominal obesity and the metabolic syndrome: contribution to global cardiometabolic risk. Arterioscler Thromb Vasc Biol 28 , 1039-1049.

29. Ekelund U, Besson H, Luan J et al. (2011) Physical activity and gain in abdominal adiposity and body weight: prospective cohort study in 288,498 men and women. Am J Clin Nutr 93, 826-835.

30. Koh-Banerjee P, Chu NF, Spiegelman D et al. (2003) Prospective study of the association of changes in dietary intake, physical activity, alcohol consumption, and smoking with $9-y$ gain in waist circumference among 16587 US men. Am J Clin Nutr 78, 719-727.

31. Sherwood NE, Jeffery RW, French SA et al. (2000) Predictors of weight gain in the Pound of Prevention study. Int J Obes Relat Metab Disord 24, 395-403.

32. Mosca CL, Marshall JA, Grunwald GK et al. (2004) Insulin resistance as a modifier of the relationship between dietary fat intake and weight gain. Int J Obes Relat Metab Disord 28, 803-812.

33. Hu FB, Li TY, Colditz GA et al. (2003) Television watching and other sedentary behaviors in relation to risk of obesity and type 2 diabetes mellitus in women. JAMA 289, 1785-1791.

34. Halkjaer J, Sorensen TI, Tjonneland A et al. (2004) Food and drinking patterns as predictors of 6-year BMI-adjusted changes in waist circumference. Br J Nutr 92, 735-748.

35. Newby PK, Muller D, Hallfrisch J et al. (2003) Dietary patterns and changes in body mass index and waist circumference in adults. Am J Clin Nutr 77, 1417-1425.
36. Vainio H \& Bianchini F (editors) (2002) IARC Handbooks of Cancer Prevention. vol. 6: Weight Control and Physical Activity. Lyon: IARC Press.

37. Seidell JC (1998) Dietary fat and obesity: an epidemiologic perspective. Am J Clin Nutr 67, 3 Suppl., 546S-550S.

38. Colditz GA, Willett WC, Stampfer MJ et al. (1990) Patterns of weight change and their relation to diet in a cohort of healthy women. Am J Clin Nutr 51, 1100-1105.

39. Ludwig DS, Pereira MA, Kroenke CH et al. (1999) Dietary fiber, weight gain, and cardiovascular disease risk factors in young adults. JAMA 282, 1539-1546.

40. Coakley EH, Rimm EB, Colditz G et al. (1998) Predictors of weight change in men: results from the Health Professionals Follow-up Study. Int J Obes Relat Metab Disord 22, 89-96.

41. Paddon-Jones D, Westman E, Mattes RD et al. (2008) Protein, weight management, and satiety. Am J Clin Nutr 87, issue 5, 1558S-1561S.

42. Halkjaer J, Tjonneland A, Thomsen BL et al. (2006) Intake of macronutrients as predictors of $5-\mathrm{y}$ changes in waist circumference. Am J Clin Nutr 84, 789-797.

43. National Health and Medical Research Council (2003) Food for Health: Dietary Guidelines for Australian Adults. Canberra: Commonwealth of Australia.

44. Department of Health and Aged Care (1999) National Physical Activity Guidelines for Adults. Canberra: Australian Government.

45. National Health and Medical Research Council (2009) Australian Guidelines to Reduce Health Risks from Drinking Alcohol. Canberra: Commonwealth of Australia. 